\title{
Retrograde amnesia for extinction: Similarities with amnesia for original acquisition memories
}

\author{
James F. BRiggs ANd DaVId C. RicCio \\ Kent State University, Kent, Ohio
}

\begin{abstract}
Two experiments were conducted using rats to determine whether extinction is susceptible to a traditional amnestic agent (i.e., hypothermia) and to examine whether amnesia for extinction follows the same characteristics as those that occur with original memories. In Experiment 1, rats received hypothermia immediately, 60 min, or 120 min after extinction. When tested, the subjects cooled shortly after extinction showed little memory of the extinction training. This amnesia for extinction disappeared with longer postextinction delays, demonstrating a temporal gradient. Experiment 2 replicated the basic finding and demonstrated that an amnestic-extinguished memory could be recovered by reexposing the subjects to the amnestic agent and that the recovered extinction memory did not persist. These findings provide more evidence that extinction is a form of new learning and are consistent with retrograde amnesia research for original memories showing a temporal gradient and alleviation of retrograde amnesia by reexposure to the amnestic agent.
\end{abstract}

Experimentally induced retrograde amnesia has been used to study memory processes in animals for many years (Spear \& Riccio, 1994). This type of amnesia has been shown using a number of traditional learning paradigms, including appetitive tasks (Andrade, Udaya, \& Chandra, 1995; Essman, 1965; Jaffe \& Blanco, 1994; Pinel, 1969; Tenen, 1965) and fear-conditioning paradigms that include conditioned emotional responses (or conditioned suppression; Avis \& Carlton, 1968; Hughes, 1969; Kohlenberg \& Trabasso, 1968; McIntyre, 1979; Nachman, Meinecke, \& Baumbach, 1969; Palfai \& Albala, 1976), conditioned taste aversion (Ahlers \& Best, 1972; Kral \& Beggerly, 1973), and both active avoidance (Duncan, 1949; Flood, Jarvik, Bennett, Orme, \& Rosenzweig, 1977; Lewis, Miller, Misanin, \& Richter, 1967) and passive avoidance (Hinderliter, Webster, \& Riccio, 1975; Madsen \& McGaugh, 1961; Riccio, Hodges, \& Randall, 1968). Thus, there has been extensive research on the characteristics of retrograde amnesia, but almost all of it has involved learning paradigms that use a reinforcer, either appetitive or aversive in nature.

In contrast, little research has been done to investigate retrograde amnesia for a learning event that does not involve explicit reinforcement. Extinction provides an example of this type of learning. In extinction, the cues previously paired with a biologically relevant reinforcer (e.g., food reward or shock) are presented without that outcome, leading to a reduction in responding.

There is a considerable amount of work showing that extinction is a process that involves new learning or memories, rather than "unlearning" or forgetting of the original learning episode. Pavlov (1927) was the first to demonstrate that an extinguished response can recover, spontaneously, over time. Because the response returned simply with the passage of time, he concluded that extinction is a form of inhibition, rather than a destruction of the response. More recently, Bouton (1993, 2002, 2004; see also Bouton \& Swartzentruber, 1991) has done extensive work further elaborating the view that extinction is not a breakdown of original association but, instead, involves new learning. Bouton described at least four phenomena (spontaneous recovery, the renewal effect, the reinstatement effect, and rapid reacquisition) that demonstrate that extinction does not destroy original learning.

If extinction is viewed as new learning, as Bouton and others have argued, manipulations that affect acquisition should also modulate extinction. Several findings seem to support this prediction. For example, Falls, Miserendino, and Davis (1992) reported that an NMDA antagonist D,L2-amino-5-phosphonovaleric acid (AP5) blocked extinction, just as it also impairs original learning. More recently, Berman and Dudai (2001) have reported that anisomycin also blocks extinction in a conditioned taste aversion paradigm. Using a double dissociation paradigm, Dudai and colleagues (Bahar, Samuel, Hazvi, \& Dudai, 2003) showed that the neural circuits underlying acquisition and extinction are not identical. In their study, anisomycin injected into the central nucleus of the amygdala impaired conditioned taste aversion learning, whereas similar infusions into the basolateral nuclei (BLA) had no effect; conversely, anisomycin administered to the BLA slowed extinction but had no effect on extinction when infused into the central nucleus (CeA).

Although these experiments are important in determining the neurobiological characteristics of extinction, the

D. C. Riccio, driccio@kent.edu 
anterograde nature of the treatments makes it difficult to know whether the impairments were due to the effects of the agent on sensory, motivational, or associative processes or whether they represented memory loss for the extinction experience.

A different approach, in which the agent is administered after extinction, suggests that memory loss is involved in the impairment of performance and strengthens the argument that extinction is new learning. For example, Vianna, Szapiro, McGaugh, Medina, and Izquierdo (2001) reported that retrograde amnesia for extinction was induced with the protein synthesis inhibitor anisomycin. In their study, rats were trained on an inhibitory avoidance task in which they were placed on a platform and received a footshock upon stepping down. The rats were subsequently tested for retention each day for 4 days. Testing served as extinction trials, because no footshocks were delivered during testing. Extinction was inferred from the shortening of step-down latencies over trials. However, amnesia for extinction was found if the rats received anisomycin infused into the CA1 area of the hippocampus after the first test (extinction) session. Step-down latencies were longer on the second test session, due to forgetting of extinction. The phenomenon of retroactively impairing extinction has been extended to medaka fish, using the amnestic agent 3-aminobenzoic acid ethyl (MS222, a sodium channel blocker; Eisenberg, Kobilo, Berman, \& Dudai, 2003). In this study, medaka fish were trained to fear a conditioned stimulus (light) that was repeatedly paired with mild electric shocks that were produced through the water. This fear conditioning produced an altered (increased) locomotion behavior to the presentation of the light. The fear response was extinguished by repeatedly presenting the light without the shock. However, when MS222 was administered by bath application immediately following the extinction trials, a decrement in performance did not occur. Thus, fish that received the sodium channel blocker showed an increase in locomotion (i.e., more fear), as compared with the control fish, because extinction was not blocked.

Although the evidence reviewed above suggests that extinction is new learning and subject to amnesia, it is not known to what extent amnesia for extinction shares the characteristics associated with amnesia for original learning. In order to facilitate comparison with the body of research on retrograde amnesia for new memory, and potentially to extend the generality of previous findings, we chose to use hypothermia, an amnestic treatment that has proven effective in many studies (Hinderliter et al., 1975; Mactutus \& Riccio, 1978; Mactutus, Riccio, \& Ferek, 1979; Misanin \& Hoover, 1971; Riccio et al., 1968). Therefore, one aim of the following experiments was to determine whether extinction is susceptible to retrograde amnesia, using hypothermia. In addition, we investigated whether any memory impairment would follow the characteristic temporal gradient and whether the amnesia could be reversed (i.e., whether the amnestic extinction memory could be recovered).

\section{EXPERIMENT 1}

One characteristic of retrograde amnesia for an original acquisition memory is its time-dependent nature, in which memory loss is inversely related to the time between the learning episode and the amnesic treatment (e.g., Duncan, 1949; Judge \& Quartermain, 1982; Mactutus, Ferek, \& Riccio, 1980; Palfai \& Chillag, 1971; Paolino, Quartermain, \& Miller, 1966; Pinel, 1969; Riccio et al., 1968; Zhou \& Riccio, 1995). If retrograde amnesia for extinction shares characteristics similar to those of original learning, there should be a reduction in memory impairment as the delay between the extinction session and the hypothermia treatment increases; that is, extinction should be observed, in that fear levels will decrease. A temporal gradient will also rule out any nonspecific stress effects or proactive effects produced by the hypothermia. The rationale here is that if the hypothermia itself alters test performance, both the short and the long delays between extinction and hypothermia should yield similar effects. In fact, any performance deficit should be more pronounced for those in the long delay condition, because the hypothermia occurs closer in time to testing, as compared with those receiving the treatment immediately following extinction.

\section{Method}

Subjects. Sixty experimentally naive, adult male Long-Evans hooded rats, obtained from Kent State University's animal facility breeding colony, served as subjects. The subjects were approximately 80 days old at the start of the experiment. The rats were housed singly, with free access to food and water, and were maintained on a 15:9-h light:dark cycle. All the experimental sessions took place during the light portion of the photocycle and at the same time each day.

Apparatus. Training, extinction, and testing were conducted in a $43 \times 18 \times 18 \mathrm{~cm}$ black-white shuttlebox with a grid floor (2-mm grids spaced $1 \mathrm{~cm}$ apart center to center). The shuttlebox was made of Plexiglas and was divided into two compartments of equal size by a guillotine door. The white compartment had a transparent Plexiglas lid, and the black compartment had an opaque black Plexiglas lid. Footshocks were delivered through the grid floor via a constant current AC shock generator (Model 5806, Lafayette Instruments, Lafayette, IN). The apparatus was located in a $2 \times 3 \mathrm{~m}$ room with white walls. This room was illuminated by a $15-\mathrm{W}$ light bulb suspended $1 \mathrm{~m}$ above the shuttlebox. White noise $(76 \mathrm{~dB})$ was presented at all times in this context in order to mask extraneous noises. The shuttlebox was situated on a round metal table located at one end of the room. A Model 2095 Forma Temp. Jr. bath and circulator containing water maintained at $4^{\circ} \mathrm{C}$ was used to produce hypothermia. Rectal temperatures were taken with a Fisher Scientific Traceable digital thermometer inserted approximately $3.2 \mathrm{~cm}$ into the rat's rectum.

Procedure. Prior to the beginning of the experiment, all the rats were handled for approximately 4 min on 2 consecutive days before being weighed and randomly assigned to one of six groups, in each of which the rats received a single punishment training trial. During training, each rat was placed in the white compartment of the black-white shuttlebox facing away from the closed guillotine door. After $15 \mathrm{sec}$, the guillotine door was raised, and the latency to cross into the black compartment (all four paws) was recorded. The door was then lowered, and two inescapable footshocks $(1 \mathrm{sec}, 0.5 \mathrm{~mA})$ were delivered 5 and $10 \mathrm{sec}$ after the door was lowered. Five seconds 
after the second footshock, the animal was removed from the apparatus and returned to its home cage. This fear conditioning produced fear of the black compartment.

Twenty-four hours after being trained, five groups received a single extinction trial. In this session, the rats were placed in the white compartment of the shuttlebox for $15 \mathrm{sec}$, then in the black compartment for $12 \mathrm{~min}$. The rats were not able to cross between compartments, since the guillotine door was lowered at all times. The parameters chosen for extinction were similar to those in previous studies (Harrod, Metzger, \& Riccio, 1996; Hinderliter \& Riccio, 1977; Morgan \& Riccio, 1994). A fear control group (No Ext) did not receive extinction or hypothermia but was tested to evaluate the level of fear without any treatment. To assess the level of extinction, a control group (Ext) received the extinction exposure but did not receive hypothermia. The design of the experiment is summarized in Table 1.

Following the extinction session, three experimental hypothermia groups (Hypo-0, Hypo-30, and Hypo-60, respectively) received the amnesic treatment (hypothermia) immediately, $30 \mathrm{~min}$, or $60 \mathrm{~min}$ after extinction to assess a temporal gradient. The hypothermia treatment consisted of restraining the rat in a clear Plexiglas perforated cylinder immersed in cold water to the neck until its colonic temperature dropped to $21^{\circ} \pm 1^{\circ} \mathrm{C}$ (a rat's normal body temperature is $37^{\circ} \mathrm{C}$ ). Rectal temperatures were checked approximately $10 \mathrm{~min}$ after immersion. (Similar procedures have been demonstrated to produce amnesia for conditioned fear; see Hinderliter \& Riccio, 1977; Hinderliter et al., 1975; Morgan \& Riccio, 1994.) Upon removal from the cold bath, the rat was dried extensively with terrycloth towels to remove all moisture from its fur and was returned to its home cage.

To assess the contribution of restraint and sudden cold exposure on performance, a stress control group (Brief Hypo) received fear conditioning, extinction, and a brief hypothermia session. This stress control group was treated identically to the immediate hypothermia group, except that the exposure to the cold water lasted only $1 \mathrm{~min}$. In a separate pilot study, a restraint control group demonstrated that restraint alone did not act as a punisher to produce fear to the black compartment or that the restraint acted as a disinhibitor from extinction. This Brief Hypo group also controlled for the possibility that exposure to the cold water bath (a novel event) acted as a disinhibitor to produce recovery from extinction. These control procedures had been used for hypothermia-induced retrograde amnesia for a passive avoidance response in the rat (Riccio et al., 1968) but needed to be replicated in examining retrograde amnesia for extinction, because the effect of hypothermia on original fear conditioning and on extinction might differ.

Twenty-four hours after the extinction session and $48 \mathrm{~h}$ after training for the fear control group, all the groups underwent passive avoidance testing. Test trials were conducted identically to training trials, except that no shocks were delivered upon the rat's entering the black compartment and the guillotine door remained open. Testing lasted for $10 \mathrm{~min}$ once the guillotine door had been opened. The rat was allowed to choose between the white and the black compartments. Latency to cross to the black side of the shuttlebox and the total time spent (TTS) on the safe (white) side were recorded as the dependent measures. Since the patterns of results were generally similar on both measures, only the TTS scores will be reported. All time measurements were recorded by a standard stopwatch.

\section{Results}

Training. The rats in all six groups exhibited short cross-latencies at training, with group means ranging from 6.8 to $8.4 \mathrm{sec}$. An ANOVA revealed no differences between the six groups $[F(5,42)=0.30, p>.50]$; thus, all the subjects exhibited similar cross-latencies at training.

Testing. Figure 1 shows the mean TTS scores for all six groups at testing. As can be seen, the No-Ext fear group had a spatial avoidance score (long TTS) dissimilar to that for the Ext group. In addition, the performance of the Hypo-0 group was similar to that of the No-Ext group and greater than that of the Ext group. The group that received the hypothermia treatment 30 min after extinction (Hypo-30) also showed as much avoidance as the Hypo-0 group. More important, the avoidance in the group receiving hypothermia $60 \mathrm{~min}$ after the extinction session (Hypo-60) was comparable to that of the Ext group. This demonstrates that the effect of hypothermia following extinction is time dependent - that is, less impairment of extinction as the extinction to hypothermia treatment delay increases. Finally, the Brief Hypo control group showed that recovery of avoidance seen in the Hypo-0 group did not result from punishment of the restraint or the sudden cold exposure following extinction.

These results were confirmed by an ANOVA that revealed an overall significant difference between groups $[F(5,42)=33, p<.001]$. Tukey post hoc tests confirmed that the Ext group produced less avoidance than did the No-Ext group $(p<.001)$. Post hoc tests also confirmed that the Hypo-0 group showed as much avoidance as the No-Ext group $(p>.50)$, as well as significantly more avoidance than the Ext group $(p<.001)$, demonstrating retrograde amnesia for extinction. Tukey's post hoc tests also revealed that the group receiving hypothermia $30 \mathrm{~min}$ after extinction (Hypo-30) showed avoidance scores comparable to those for the Hypo-0 group and the No-Ext group ( $p \mathrm{~s}>.50)$. The temporal gradient was confirmed by post hoc tests revealing that the group receiving the hypothermia treatment 60 min after extinction (Ext-60) had significantly less avoidance than did both the Hypo-0 group and the Hypo-30 group $(p$ s $<.001)$. The TTS score for the Ext-60 group did not differ significantly from

Table 1

Design for Experiment 1

\begin{tabular}{lccccc}
\hline \multicolumn{1}{c}{ Group } & Training & Extinction & Delay $(\mathrm{min})$ & $\begin{array}{c}\text { Amnesia } \\
\text { Treatment }\end{array}$ & Test \\
\hline No Ext & yes & no & - & no & yes \\
Ext & yes & yes & - & no & yes \\
Hypo-0 & yes & yes & 0 & yes & yes \\
Hypo-30 & yes & yes & 30 & yes & yes \\
Hypo-60 & yes & yes & 60 & yes & yes \\
Brief Hypo & yes & yes & 0 & brief & yes \\
\hline
\end{tabular}

Note-Ext, extinction; Hypo, hypothermia. The time between training and extinction and between amnesia treatment and test was $24 \mathrm{~h}$. 


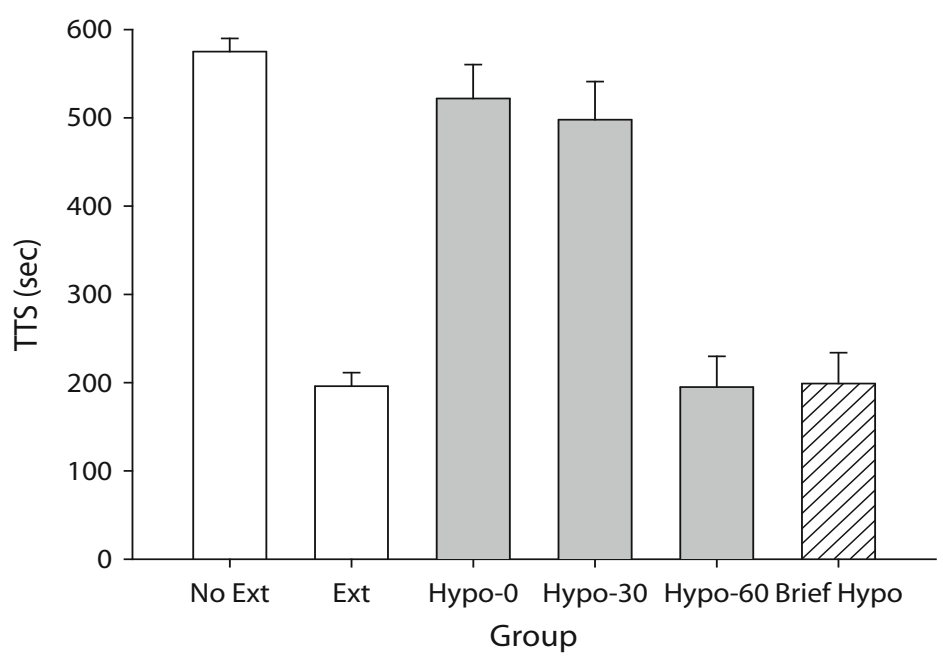

Figure 1. Mean ( $+S E M)$ total time spent (TTS, in seconds) on the safe (white) side of the black-white shuttlebox for all the groups in Experiment 1. Open bars represent the no-extinction and extinction control groups. Shaded bars represent experimental groups. Increased TTS scores for the group that received hypothermia immediately following extinction (Hypo-0), as compared with the extinction group (Ext), represents retrograde amnesia for extinction. Group Hypo-60 demonstrates that with longer extinction to hypothermia delays, the retrograde amnesia for extinction is attenuated (i.e., temporal gradient). Group Brief Hypo (shaded slashed bar) shows that the brief hypothermia treatment had no effect on the retrograde amnesia for extinction.

that for the Ext group $(p>.50)$. Confirming that stress from the restraint or from exposure to the cold water did not cause fear at test, the post hoc tests revealed that the avoidance performance in the Brief Hypo group failed to differ from that of the Ext group $(p>.50)$ and was significantly lower than that of the Hypo-0 group $(p<.001)$. Thus, retrograde amnesia for extinction was not caused by the brief hypothermia treatment.

\section{Discussion}

The results indicate that extinction is susceptible to retrograde amnesia produced by hypothermia. Moreover, the amnesia is time dependent and is not simply a general impairment of performance. This outcome is consistent with the view that extinction is a form of new learning. More specifically, these results extend previous findings that extinction memories are similar to original acquisition memories by showing that memories for extinction are vulnerable shortly after learning (while active or during information processing) but are less susceptible to retrograde amnesia with longer postextinction delays; that is, longer extinction to hypothermia delays produced less retrograde amnesia.

Since novel stimuli can produce release from extinction, or disinhibition, an additional control group that received a brief exposure to the cold water bath was included. This control condition demonstrated that the stress from exposure to the cold water alone did not produce the increased fear shown by the Hypo-0 group or by the Hypo-30 group. Similarly, the Hypo-60 group provided another demonstra- tion that the hypothermia treatment itself did not result in a change in performance. Although this long-delay group received the same treatment as the other hypothermia groups, avoidance of the fear compartment was similar to that for the Ext group and the stress control group.

These results, showing a time-dependent feature of retrograde amnesia for extinction, can be accounted for by using a retrieval-oriented interpretation of retrograde amnesia that invokes a postacquisition state-dependent effect (Millin, Moody, \& Riccio, 2001; Riccio, Millin, \& Gisquet-Verrier, 2003; Riccio, Moody, \& Millin, 2002; see also Hinderliter et al., 1975). According to this retrieval view, there is a short period of time following training when the learned information continues to be processed. If an amnestic agent is presented during this processing, the information becomes encoded with the altered internal context produced by the amnestic agent. Thus, the longer the interval between the training episode and the amnestic treatment, the more likely it is that the memory will be encoded within the normal context, resulting in the characteristic temporal gradient of retrograde amnesia.

The hypothermia treatment administered either immediately following or $30 \mathrm{~min}$ after the extinction session can be viewed as resulting in retrograde amnesia for extinction, since the extinction memory was encoded in a $\mathrm{cool}$ state and testing was in the normal temperature state. However, when hypothermia was administered $60 \mathrm{~min}$ after the extinction session, the memory would have been encoded in the normal body temperature context, resulting in the expression of extinction at testing. 


\section{EXPERIMENT 2}

The results of Experiment 1 demonstrated retrograde amnesia for extinction and showed also that retrograde amnesia for extinction (like retrograde amnesia for acquisition) followed a temporal gradient. In the second experiment, we investigated further the similarities between retrograde amnesia for extinction and retrograde amnesia for an original training episode. In addition, this experiment served to examine the issue of a retrieval failure view versus a storage failure account of retrograde amnesia. The retrieval failure notion views memory loss as the inability to access (or retrieve) information that has been stored (Spear, 1978), whereas the consolidation view describes forgetting as an interruption in the storage (or consolidation) of the information.

As has been stated above, retrograde amnesia can be attenuated by reexposing the subject to the amnesic agent prior to testing (Bradley \& Galal, 1988; Hinderliter et al., 1975; Mactutus \& Riccio, 1978; Thompson \& Neely, 1970). This reversibility of retrograde amnesia by reexposing the subjects to the amnestic agent favors a retrievalbased account of retrograde amnesia (see Millin et al., 2001; Riccio, Millin, \& Bogart, 2006; Riccio et al., 2003). According to this retrieval view, if the amnestic agent (hypothermia) is administered during the processing of the learned event, the information becomes encoded with an altered internal context (i.e., a cool state). If tested while at a normal body temperature, there will be a mismatch in cues, leading to the failure of memory retrieval. However, reexposure to the amnestic agent (hypothermia) before testing will return the internal context to the cool state, leading to the alleviation of retrograde amnesia. Thus, in Experiment 2, the subjects were recooled prior to testing in order to assess whether retrograde amnesia was alleviated by returning rats to the internal context present immediately following extinction.

If body temperature is important, as we have suggested, one might expect the amnesia for extinction to reappear after the rats' temperature returns to normal levels. A second aim of Experiment 2 was to test this prediction by incorporating a delay between the reexposure to the deepbody cooling and testing. Again, following the retrieval account of retrograde amnesia, when the rats rewarm, the adequate retrieval cues should not be available. Thus, amnesia for the extinction memory should again be evident.

\section{Method}

Subjects and Apparatus. Sixty experimentally naive, adult male Long-Evans hooded rats were subjects. The animals were obtained and maintained as in Experiment 1. Prior to experimental manipulations, all the rats were treated as in Experiment 1. The apparatus was the same as that used in Experiment 1.

Procedure. All handling, training, extinction, hypothermia, and testing were identical to those in Experiment 1, except where noted.

The rats were randomly assigned to one of six groups before training. Twenty-four hours after being trained, five groups received extinction. One fear control group (No Ext) did not receive extinction, hypothermia, or recooling but was tested merely to determine the level of fear without any treatment. One extinction control group (Ext) received extinction but did not receive hypothermia or recooling and was tested to evaluate the level of extinction. The design of the experiment is summarized in Table 2 .

Immediately following extinction, four groups received the amnesic treatment (hypothermia). Twenty-four hours after extinction and hypothermia, three reexposed experimental groups received a second hypothermia treatment, similar to the first one, in order to reactivate the memory of extinction. These subjects were recooled to $25^{\circ} \pm 1^{\circ} \mathrm{C}$. As their body temperature rose, one group (Recool 30 ) was tested at $30^{\circ} \mathrm{C}$, one group (Recool 33 ) at $33^{\circ} \mathrm{C}$, and another group (Recool 37$)$ at $37^{\circ} \mathrm{C}$. A hypothermia control group (Ext/Hypo) received training, extinction, and hypothermia, but no recooling, to replicate retrograde amnesia for extinction as in Experiment 1. Testing was conducted as described in Experiment 1.

\section{Results}

Training. The rats in all six groups exhibited short cross-latencies at training, with group means ranging from 7.1 to $8.8 \mathrm{sec}$. An ANOVA revealed no differences between the six groups $[F(5,42)=0.37, p>.50]$; thus, all the subjects exhibited similar cross-latencies at training.

Testing. Figure 2 shows the mean TTS scores for all six groups at testing. As can be seen, the No-Ext fear group has a spatial avoidance score (long TTS) dissimilar to that for the Ext group. Furthermore, the performance of the Ext/Hypo group was similar to that of the No-Ext group and was greater than that of the Ext group, again demonstrating that the hypothermia treatment produced retrograde amnesia for extinction. More important, the group that received the second hypothermia treatment and was tested at a low body temperature of $30^{\circ} \mathrm{C}$ (the Recool 30 group) showed as much avoidance as the Ext group. In addition, the group that was recooled and tested at $33^{\circ} \mathrm{C}$ (Recool 33) had a slight increase in avoidance scores but showed spatial avoidance similar to that for the Recool 30 and Ext groups. The Recool 37 group demonstrated that

Table 2

Design for Experiment 2

\begin{tabular}{lccccc}
\hline Group & Training & Extinction & $\begin{array}{c}\text { Amnesia } \\
\text { Treatment }\end{array}$ & $\begin{array}{c}\text { Amnesia } \\
\text { Treatment }\end{array}$ & $\begin{array}{c}\text { Test/ } \\
\text { Temperature }\end{array}$ \\
\hline No Ext & yes & no & no & no & yes/- \\
Ext & yes & yes & no & no & yes/- \\
Ext/Hypo & yes & yes & yes & no & yes $/-$ \\
Recool 30 & yes & yes & yes & yes & yes $/ 30^{\circ}$ \\
Recool 33 & yes & yes & yes & yes & yes $/ 33^{\circ}$ \\
Recool 37 & yes & yes & yes & yes & yes $/ 37^{\circ}$ \\
\hline
\end{tabular}

Note-Ext, extinction; Hypo, hypothermia. The time between training and extinction and between the first and second amnesia treatments was $24 \mathrm{~h}$. 


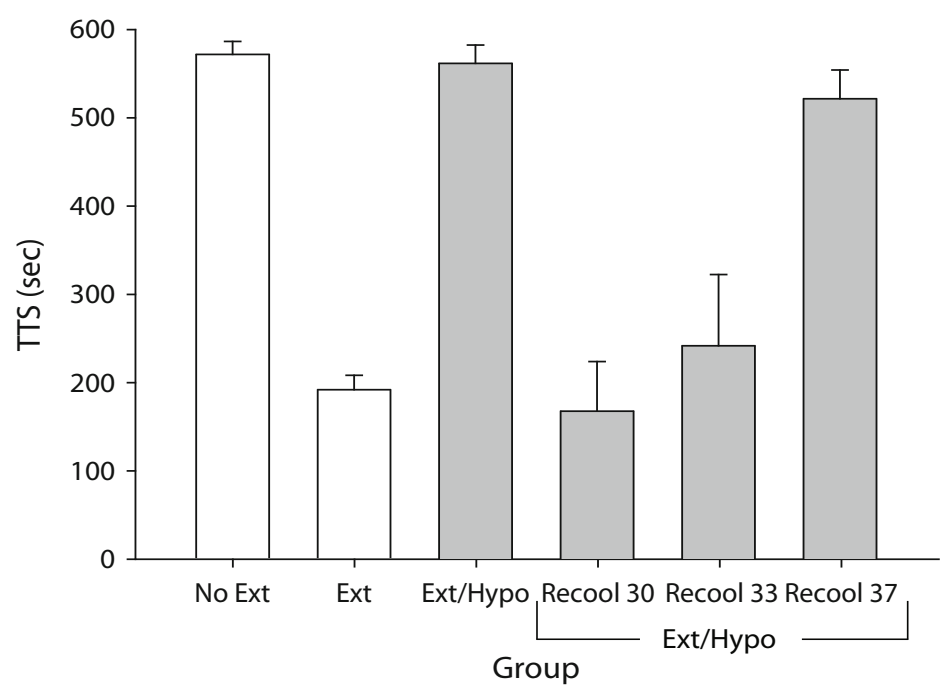

Figure 2. Mean $(+S E M)$ total time spent (TTS, in seconds) on the safe (white) side of the black-white shuttlebox for all the groups in Experiment 2. Open bars represent the no-extinction and extinction control groups. Shaded bars represent experimental groups. Groups within the "Ext/Hypo" bracket received extinction followed by hypothermia before being recooled. Increased TTS scores for the group that received hypothermia immediately following extinction (Ext/Hypo), as compared with the extinction group (Ext), represents retrograde amnesia for extinction. The group that was reexposed to the hypothermia before test (Recool 30) and tested at a lower body temperature demonstrates that retrograde amnesia for extinction can be alleviated. Group Recool 37 shows that this recovered amnestic extinction memory does not persist but, rather, depends on body temperature.

amnestic extinction memory is not permanently reestablished by showing avoidance scores comparable to those for both the No-Ext group and the Ext/Hypo group. The mean times to reach the target temperatures for the Recool 30, Recool 33, and Recool 37 groups were $47 \mathrm{~min}$ $(S D=6.2), 68 \mathrm{~min}(S D=11.5)$, and $99 \min (S D=$ 15.0), respectively.

The behavioral results were confirmed by an ANOVA that revealed an overall significant difference between groups $[F(5,42)=19.5, p<.001]$. Tukey post hoc tests confirmed that the Ext group produced less avoidance than did the No-Ext group $(p<.001)$. Post hoc tests also confirmed that the Ext/Hypo group showed as much avoidance as did the No-Ext group $(p>.50)$, as well as significantly more avoidance than did the Ext group $(p<$ .001 ), again replicating retrograde amnesia for extinction. Tukey's post hoc tests also confirmed the attenuation of retrograde amnesia for extinction by revealing that the group that was reexposed to the hypothermia treatment and was tested at a cool body temperature (Recool 30) showed spatial avoidance scores comparable to those for the Ext group $(p>.50)$. Group Recool 33 also showed a level of avoidance similar to those for both the Ext and the Recool 30 groups $(p>.50)$. The group that was recooled and allowed to rewarm to approximately normal body temperature (Recool 37) demonstrated that the reactivated extinction memory returns to an amnesic state by showing as much spatial avoidance as did the No-Ext group $(p>$ $.50)$ and the Hypo/Ext group $(p>.50)$.

\section{Discussion}

Experiment 2 confirmed that extinction is susceptible to retrograde amnesia produced by hypothermia. This experiment also demonstrated that reexposure to the amnestic agent attenuates retrograde amnesia for an extinguished memory: Rats exposed to a second hypothermic treatment before testing performed as if the extinction memory was reactivated; that is, the amnestic memory was recovered. This outcome is similar to that obtained with retrograde amnesia for original acquisition and provides further novel evidence that extinction represents another form of learning.

As is true for original learning, the recovery of the amnestic extinction memory was not permanently reestablished. If the rats were allowed to warm to normal body temperature, the amnestic extinction memory was again unavailable; that is, the extinction memory was again forgotten. This finding is consistent with work investigating reactivation of an original amnestic memory by reexposure to hypothermia (Hinderliter et al., 1975). Hinderliter and colleagues found that an amnestic fear memory was retrieved following a second hypothermia treatment before test but that this recovered memory did not persist when the rats were tested $4 \mathrm{~h}$ after recooling. Although the present experiment utilized an amnestic memory for extinction, the results were similar to those of original learning, in that the recovered amnestic extinction memory did not persist when the cues associated with the memory were no longer available. 
The retrieval account of retrograde amnesia described above postulates an encoding-retrieval mismatch that appears to work also with extinction learning. We assume that following the extinction session, the information continues to become encoded. When the animal is made hypothermic shortly after extinction, during memory processing, the extinction memory is stored in a cool state. Thus, when the rat is tested while at normal body temperature, the appropriate retrieval cues are not available for memory retrieval (Experiments 1 and 2). However, if the rat is reexposed to the amnestic agent before testing, the cool cues are restored, which allows for retrieval of the extinction memory (Experiment 2). Conversely, the animals that were allowed to rewarm to normal body temperature following the second hypothermia treatment did not have the appropriate cues available for memory retrieval of extinction, and so the extinction memory again became inaccessible; that is, the conditioned fear response was retrieved.

The preceding experiments suggest that retrograde amnesia for extinction can be explained by invoking a statedependent model in which the nonreinforced exposure becomes encoded in an internal cool state produced by hypothermia. Thus, when tested at normal body temperature, the appropriate cues are not available for adequate retrieval. There is essentially a shift in the internal contexts. Conceptually, this account of amnesia for extinction seems similar to Bouton's (Bouton \& Bolles, 1979; Bouton \& King, 1983) renewal effect, which demonstrates that extinction is context specific. Renewal is observed when there is a change in context after extinction, causing a return of the original response. The original learning returns (renewal) because of the lack of extinction retrieval cues available. Thus, extinction is specific to the context in which the learning takes place. Indeed, research from our lab has also demonstrated that exposure to the original training context immediately following extinction in a different context provides the appropriate external retrieval cues to alleviate the renewal effect (Briggs, 2006).

The state-dependent account of retrograde amnesia is similar to the renewal effect if the internal state is considered to be a different context. For example, in the experiments described above, hypothermia shortly after extinction presumably encoded the memory of nonreinforcement in a cool state, and consequently, the information was unavailable when the rats were tested with a normal body temperature.

One caveat here is that the rewarming to different target temperatures is confounded with time. As was stated earlier, the mean times to reach the target temperatures differed for the Recool 30, Recool 33, and Recool 37 groups. Although it was assumed that the body temperature at the time of testing was the critical factor-that is, the appropriate $\mathrm{cool}$ cues were not available if the rat was allowed to rewarm to normal body temperature- perhaps the recovery from amnesia is simply time limited. We think the latter interpretation is unlikely, since in another study on recovery of a conditioned response, Mactutus, McCutcheon, and Riccio (1980) demonstrated that temperature, rather than time, was the critical variable. In their study, rats were exposed to warm water following a hypothermic reactivation treatment, in order to reverse the hypothermia. In this case, even when the rats were tested at the shortest interval, the retrograde amnesia remained present, presumably because the appropriate cool cues were unavailable. This led the authors to conclude that "memory retrieval appears importantly linked to temperature-related contextual cues" (Mactutus, McCutcheon, \& Riccio, 1980, p. 875). The Mactutus finding strongly suggests that in the present paradigm, body temperature at testing is the critical factor, not the mere passage of time.

Although the rats were tested after being recooled, the potential complication that the results were due to cooling-induced inactivity can be ruled out. In fact, the opposite results would have been obtained if disruption in behavior had occurred due to reexposure to the hypothermic treatment. The passive avoidance test used here asks the animals to remember the initial training; thus, good performance results in avoiding the black compartment. If recooling resulted in inactivity, the rats that were recooled and tested shortly following the cold water exposure (Recool 30) should not have crossed into the unsafe side. However, the behavior of this group was similar to that of the group that received only the extinction treatment; that is, they crossed into the black compartment. Thus, being recooled shortly before testing did not result in a change in activity that would account for the outcome.

\section{GENERAL DISCUSSION}

The present findings demonstrate that exposure to an amnestic agent shortly following a brief conditioned stimulus (CS) alone exposure produces retrograde amnesia for the extinction effect. This outcome is consistent with previous reports in which extinction was blocked by treatments prior to (Bahar et al., 2003; Berman \& Dudai, 2001; Falls et al., 1992) or following (Eisenberg et al., 2003; Vianna et al., 2001) nonreinforced exposure to the CS. Our study extends the earlier work by establishing a time-dependent gradient of retrograde amnesia and by showing that the amnesia is reversible, phenomena that have been characteristic of many experiments in which amnesia for the original conditioning episode has been investigated. In addition, the effectiveness of hypothermia seen here extends the generality of the kinds of treatments that produce retrograde amnesia for extinction.

It is worth noting that the reversibility of retrograde amnesia for extinction (i.e., the recovery of the original training) obtained by reexposing the subjects to the amnestic agent is consistent with a retrieval-oriented interpretation of retrograde amnesia (Millin et al., 2001; Riccio et al., 2003; Riccio et al., 2002; see also Hinderliter et al., 1975). According to this view, the extinction learning continues to be processed during the cool state produced by the hypothermia treatment, resulting in the extinction memory's becoming encoded in the altered state. If hypothermia is administered following a delay after extinction, there is no amnesia for extinction, because the processing of information was completed before the cooling. Recovery of memory would be accounted for with similar processes: 
If the body temperature is again reduced by reexposure to hypothermia, the appropriate cool cues are available, and the amnestic extinction memory is retrieved. Furthermore, if the animals are allowed to rewarm, the appropriate cues are no longer available, and the extinction memory cannot be accessed.

Although retrograde amnesia is typically a time-limited phenomenon, an important exception has been demonstrated: If an old memory is reactivated by a brief exposure to some aspect of the training episode just prior to the amnestic treatment, the memory can again become susceptible to retrograde amnesia (Judge \& Quartermain, 1982; Mactutus et al., 1979; Misanin, Miller, \& Lewis, 1968; Nader, Schafe, \& LeDoux, 2000; Richardson, Riccio, \& Mowery, 1982; see also Millin et al., 2001). This phenomenon, often referred to as reconsolidation (but see, e.g., Riccio et al., 2006, and Sara \& Hars, 2006, for other interpretations), raises an interesting question: Given that the extinction exposures are similar to the reactivation used to induce retrograde amnesia for old memory, why did we not obtain retrograde amnesia for the original fear conditioning?

One way to conceptualize the issue is in terms of two competing processes. On the one hand, a brief extinction exposure may serve to activate the original memory but may not be sustained long enough for new learning to occur. There is substantial evidence that brief CS exposures can serve as reminders that alleviate amnesia (Gordon \& Mowrer, 1980), reduce spontaneous forgetting over time (Hamberg \& Spear, 1978), or even increase the strength of a conditioned response, as in paradoxical enhancement (Rohrbaugh \& Riccio, 1970). In such a case, the amnestic agent should act on original memory. On the other hand, more prolonged exposures afford the organism an opportunity to learn that the expected event (unconditioned stimulus) does not occur. Accordingly, the treatment should act on the memory for extinction.

Our view here is similar to the dominant trace notion proposed by Dudai (2004). For example, Eisenberg et al. (2003) have described the outcome of the competition between reconsolidation through reactivation and extinction's being vulnerable to an amnestic agent as "the trace that retains or is in the process of gaining appreciable control over behavior after the retrieval session (i.e., becomes dominant) is the one that displays transient sensitivity to the [amnestic treatment]" (p. 1104).

That the duration of exposure is important in determining the outcome obtained is supported directly by findings reported by Power, Berlau, McGaugh, and Steward (2006) and Suzuki et al. (2004). Power and colleagues demonstrated that administering the amnestic agent anisomycin after a brief reminder resulted in forgetting of the original task. If, however, anisomycin was given following a prolonged reminder session that produced extinction, the extinction learning was disrupted. Similarly, in Suzuki et al.'s study, the amount of extinction time was shown to be the important factor in determining whether the original memory or the extinction memory became susceptible to amnesia. Mice were trained to fear a particular context by receiving unsignaled footshocks while in that context. Twenty-four hours after training, the mice were divided into groups and were reexposed to the training context for 0 (no reexposure), 1, 3, or $30 \mathrm{~min}$. The different amounts of reexposure served to assess the duration at which exposure gained control over behavior - that is, a 1-min duration reactivated the memory and 30 min served as an extinction session. Twenty-four hours after reexposure, the mice were tested in the training context by measuring the percentage of time spent freezing. The results showed that being exposed to the context for 0,1 , or 3 min served as reminders but did not produce extinction. These mice showed a considerable amount of freezing. The mice that were exposed for $30 \mathrm{~min}$ demonstrated extinction (i.e., a significant decrease in freezing). However, if the amnestic agent anisomycin was administered before the 3-min reexposure session, the original training was forgotten. The protein synthesis inhibitor produced anterograde amnesia for the reactivated old memory of training. The interesting finding, which demonstrated the importance of the duration of exposure, was that if the mice were given anisomycin prior to the 30-min exposure, they forgot the extinction session, leaving the original memory intact. These mice showed as much freezing (fear) as the control groups that received the short memory reactivation sessions. Thus, if anisomycin was administered prior to the brief reexposure, the original memory was susceptible to amnesia. However, if the agent was administered before the longer exposure treatment, the extinction learning became vulnerable to the amnestic treatment.

That extinction, like original conditioning, is susceptible to retrograde amnesia is consistent with the concept that extinction is a process involving new learning (Bouton, 2002, 2004). Our finding that retrograde amnesia for extinction can be reversed provides quite compelling evidence that the original learning remained intact and that two different traces must have coexisted. Thus, amnesia for extinction can be added to the other phenomena- the renewal effect, spontaneous recovery, rapid reacquisition, and reinstatement - that Bouton has described as evidence that extinction is new learning.

\section{AUTHOR NOTE}

Funding of this research was provided by NIMH Grant 37535 to D.C.R. The authors acknowledge the helpful contributions of Pat Mlynar to this project. Correspondence should be addressed to D. C. Riccio, Department of Psychology, Kent State University, Kent, OH 44242 (e-mail: driccio@kent.edu).

\section{REFERENCES}

Ahlers, R. H., \& Best, P. J. (1972). Retrograde amnesia for discriminated taste aversions: A memory deficit. Journal of Comparative \& Physiological Psychology, 79, 371-376.

Andrade, C., Udaya, H. B., \& Chandra, J. S. (1995). BR-16A restricts development of electroconvulsive shock-induced retrograde amnesia. Biological Psychiatry, 37, 820-822.

Avis, H. H., \& CARLTON, P. L. (1968). Retrograde amnesia produced by hippocampal spreading depression. Science, 161, 73-75.

Bahar, A., Samuel, A., Hazvi, S., \& Dudai, Y. (2003). The amygdalar circuit that acquires taste aversion memory differs from the circuit that extinguishes it. European Journal of Neuroscience, 17, 1527-1530. 
Berman, D. E., \& Dudai, Y. (2001). Memory extinction, learning anew, and learning the new: Dissociations in the molecular machinery of learning in cortex. Science, 291, 2417-2419.

Bouton, M. E. (1993). Context, time, and memory retrieval in the interference paradigms of Pavlovian learning. Psychological Bulletin, 114, 80-99.

Bouton, M. E. (2002). Context, ambiguity, and unlearning: Sources of relapse after behavioral extinction. Biological Psychiatry, 52, 976-986.

Bouton, M. E. (2004). Context and behavioral processes in extinction. Learning \& Memory, 11, 485-494.

Bouton, M. E., \& Bolles, R. C. (1979). Contextual control of the extinction of conditioned fear. Learning \& Motivation, 10, 445-466.

Bouton, M. E., \& KING, D. A. (1983). Contextual control of the extinction of conditioned fear: Tests for the associative value of the context Journal of Experimental Psychology: Animal Behavioral Processes, 9, 248-265.

Bouton, M. E., \& Swartzentruber, D. (1991). Sources of relapse after extinction in Pavlovian and instrumental learning. Clinical Psychology Review, 11, 123-140.

Bradley, P. M., \& Galal, K. M. (1988). State-dependent recall can be induced by protein synthesis inhibition: Behavioral and morphological observations. Developmental Brain Research, 40, 243-251.

BRIGGS, J. F. (2006). Retrograde amnesia for extinction: Similarities with amnesia for original acquisition memories. Dissertation $A b-$ stracts International, 67(10B), 6108. (UMI No. 3236838)

DudAI, Y. (2004). The neurobiology of consolidations, or, how stable is the engram? Annual Review of Psychology, 55, 51-86.

DunCAN, C. P. (1949). The retroactive effect of electroshock on learning. Journal of Comparative \& Physiological Psychology, 42, 32-44.

Eisenberg, M., Kobilo, T., Berman, D. E., \& Dudai, Y. (2003). Stability of retrieved memory: Inverse correlation with trace dominance. Science, 301, 1102-1104.

Essman, W. B. (1965). Retrograde amnesic effect of audiogenic seizure on the reversal of a maze response. Perceptual \& Motor Skills, 20 501-504

Falls, W. A., Miserendino, M. J. D., \& Davis, M. (1992). Extinction of fear-potentiated startle: Blockade by infusion of an NMDA antagonist into the amygdala. Journal of Neuroscience, 12, 854-863.

Flood, J. F., Jarvik, M. E., Bennett, E. L., Orme, A. E., \& RosenZWEIG, M. R. (1977). Protein synthesis inhibition and memory for pole jump active avoidance and extinction. Pharmacology, Biochemistry, \& Behavior, 7, 71-77.

Gordon, W. C., \& Mowrer, R. R. (1980). An extinction trial as a reminder treatment following electroconvulsive shock. Animal Learning \& Behavior, 8, 363-367.

Hamberg, J. M., \& Spear, N. E. (1978). Alleviation of forgetting of discrimination learning. Learning \& Motivation, 9, 466-476.

Harrod, S. B., Metzger, M. M., \& Riccio, D. C. (1996). Does induced recovery from amnesia represent a disinhibition effect? Physiology \& Behavior, 60, 1375-1378.

Hinderliter, C. F., \& Riccio, D. C. (1977). Long-term effects of prior experience in attenuating amnesia. American Journal of Psychology, 90, 407-418

Hinderliter, C. F., Webster, T., \& Riccio, D. C. (1975). Amnesia induced by hypothermia as a function of treatment-test interval and recooling in rats. Animal Learning \& Behavior, 3, 257-263.

Hughes, R. A. (1969). Retrograde amnesia in rats produced by hippocampal injections of potassium chloride: Gradient of effect and recovery. Journal of Comparative \& Physiological Psychology, 68 637-644.

JAFFE, K., \& BlanCo, M. E. (1994). Involvement of amino acids, opioids, nitric oxide, and NMDA receptors in learning and memory consolidation in crickets. Pharmacology, Biochemistry, \& Behavior, 47, 493-496.

Judge, M. E., \& Quartermain, D. (1982). Characteristics of retrograde amnesia following reactivation of memory in mice. Physiology \& Behavior, 28, 585-590.

Kohlenberg, R., \& Trabasso, T. (1968). Recovery of a conditioned emotional response after one or two electroconvulsive shocks. Journal of Comparative \& Physiological Psychology, 65, 270-273.
Kral, P. A., \& Beggerly, H. D. (1973). Electroconvulsive shock impedes association formation: Conditioned taste aversion paradigm. Physiology \& Behavior, 10, 145-147.

Lewis, D. J., Miller, R. R., Misanin, J. R., \& Richter, N. G. (1967). ECS-induced retrograde amnesia for one trial active avoidance. Psychonomic Science, 8, 485-486.

Mactutus, C. F., Ferek, J. M., \& Riccio, D. C. (1980). Amnesia induced by hyperthermia: An unusually profound, yet reversible, memory loss. Behavioral \& Neural Biology, 30, 260-277.

Mactutus, C. F., McCutcheon, K., \& Riccio, D. C. (1980). Body temperature cues as contextual stimuli: Modulation of hypothermiainduced retrograde amnesia. Physiology \& Behavior, 25, 875-883.

Mactutus, C. F., \& Riccio, D. C. (1978). Hypothermia-induced retrograde amnesia: Role of body temperature in memory retrieval. Physiological Psychology, 6, 18-22.

Mactutus, C. F., Riccio, D. C., \& FereK, J. M. (1979). Retrograde amnesia for old (reactivated) memory: Some anomalous characteristics. Science, 204, 1319-1320.

Madsen, M. C., \& McGaugh, J. L. (1961). The effect of ECS on onetrial avoidance learning. Journal of Comparative \& Physiological Psychology, 54, 522-523.

MCINTYRE, D. C. (1979). Effects of focal vs generalized kindled convulsions from anterior neocortex or amygdala on CER acquisition in rats. Physiology \& Behavior, 23, 855-859.

Millin, P. M., Moody, E. W., \& Riccio, D. C. (2001). Interpretations of retrograde amnesia: Old problems redux. Nature Reviews Neuroscience, 2, 68-70.

Misanin, J. R., \& Hoover, M. (1971). Recovery rate as a determinant of the amnesic-like effect of hypothermia. Physiology \& Behavior, 6, 689-693

Misanin, J. R., Miller, R. R., \& Lewis, D. J. (1968). Retrograde amnesia produced by electroconvulsive shock after reactivation of a consolidated memory trace. Science, 160, 554-555.

Morgan, R. E., \& Riccio, D. C. (1994). Extinction of an amnestic memory in rats: Evidence for the malleability of "inaccessible" information. Learning \& Motivation, 25, 431-446.

Nachman, M., Meinecke, R. O., \& Baumbach, H. D. (1969). Temporal gradient of recovery of a conditioned emotional response following a single electroconvulsive shock. Psychonomic Science, 17, 137-138.

Nader, K., Schafe, G. E., \& LeDoux, J. E. (2000). Fear memories require protein synthesis in the amygdala for reconsolidation after retrieval. Nature, 406, 722-726.

Palfai, T., \& Albala, B. (1976). Time-dependent performance impairments produced by metrazol: Amnesia or nonspecific drug effect? Behavioral Biology, 17, 453-461.

Palfai, T., \& Chillag, D. (1971). Time-dependent memory deficits produced by pentylenetetrazol (metrazol): The effect of reinforcement magnitude. Physiology \& Behavior, 7, 439-442.

Paolino, R. M., Quartermain, D., \& Miller, N. E. (1966). Different temporal gradients of retrograde amnesia produced by carbon dioxide anesthesia and electroconvulsive shock. Journal of Comparative \& Physiological Psychology, 62, 270-274.

Pavlov, I. P. (1927). Conditioned reflexes: An investigation of the physiological activity of the cerebral cortex (G. V. Anrep, Trans.). London: Oxford University Press.

PINEL, J. P. J. (1969). A short gradient of ECS-produced amnesia in a one-trial appetitive learning situation. Journal of Comparative \& Physiological Psychology, 68, 650-655.

Power, A. E., Berlau, D. J., McGaugh, J. L., \& Steward, O. (2006) Anisomycin infused into the hippocampus fails to block "reconsolidation" but impairs extinction: The role of re-exposure duration. Learning \& Memory, 13, 27-34.

Riccio, D. C., Hodges, L. A., \& Randall, P. K. (1968). Retrograde amnesia produced by hypothermia in rats. Journal of Comparative \& Physiological Psychology, 66, 618-622.

Riccio, D. C., Millin, P. M., \& BogarT, A. R. (2006). Reconsolidation: A brief history, a retrieval view, and some recent issues. Learning \& Memory, 13, 536-544.

Riccio, D. C., Millin, P. M., \& Gisquet-Verrier, P. (2003). Retrograde amnesia: Forgetting back. Current Directions in Psychological Science, 12, 41-44. 
Riccio, D. C., Moody, E. W., \& Millin, P. M. (2002). Reconsolidation reconsidered. Integrative Physiological \& Behavioral Science, 37, 245-253.

Richardson, R., Riccio, D. C., \& Mowrey, H. (1982). Retrograde amnesia for previously acquired Pavlovian conditioning: UCS exposure as a reactivation treatment. Physiological Psychology, 10, 384-390.

Rohrbaugh, M., \& Riccio, D. C. (1970). Paradoxical enhancement of learned fear. Journal of Abnormal Psychology, 75, 210-216.

SARA, S. J., \& HARS, B. (2006). In memory of consolidation. Learning \& Memory, 13, 515-521.

SPEAR, N. E. (1978). The processing of memories: Forgetting and retention. Hillsdale, NJ: Erlbaum.

Spear, N. E., \& Riccio, D. C. (1994). Memory: Phenomena and principles. Boston: Allyn \& Bacon.

Suzuki, A., Josselyn, S. A., Frankland, P. W., Masushige, S., Silva, A. J., \& KIDA, S. (2004). Memory reconsolidation and extinction have distinct temporal and biochemical signatures. Journal of Neuroscience, 24, 4787-4795.

Tenen, S. S. (1965). Retrograde amnesia from electroconvulsive shock in a one-trial appetitive learning task. Science, 148, 1248-1250.

Thompson, C. I., \& Neely, J. E. (1970). Dissociated learning in rats produced by electroconvulsive shock. Physiology \& Behavior, 5 , 783-786.

Vianna, M. R. M., Szapiro, G., McGaugh, J. L., Medina, J. H., \& IZQUIERDO, I. (2001). Retrieval of memory for fear-motivated training initiates extinction requiring protein synthesis in the rat hippocampus Proceedings of the National Academy of Sciences, 98, 12251-12254.

ZHOU, Y., \& Riccio, D. C. (1995). Concussion-induced retrograde amnesia in rats. Physiology \& Behavior, 57, 1107-1115.

(Manuscript received February 16, 2007; revision accepted for publication May 16, 2007.) 